\title{
Contribution à l'étude de la production des taillis de Chêne vert en forêt de la Gardiole de Rians (Var)
}

\author{
Françoise MIGLIORETTI \\ Université des Sciences et Techniques de Saint-Jérôme, Laboratoire de Botanique et d'Ecologie, \\ Rue Henri-Poincaré, F 13397 Marseille Cedex 13
}

\begin{abstract}
Résumé
Cet article traite de la méthodologie mise au point pour l'étude de la production en volume et biomasse des taillis sclérophylles dans le massif forestier de la Gardiole de Rians (Var), ainsi que de la croissance en diamètre et hauteur des brins de Chêne vert en vue de dégager un certain nombre de classes de fertilité. La prise en compte de tous les brins d'une cépée y compris ceux de faible diamètre, permet de mieux exprimer la production du taillis au regard de celle définie en dendrométrie classique pour les futaies, et de parvenir ainsi à une meilleure compréhension du fonctionnement des cépées de Chêne vert qui émettent des brins à des périodicités successives, et d'aborder les problèmes de concurrence intraspécifique pour l'utilisation des ressources du milieu. Cette étude rend compte aussi des modèles mathématiques retenus pour les diverses évaluations de production ainsi que pour la croissance en diamètre et hauteur des tiges de chêne vert.
\end{abstract}

Mots clés: Croissance, production, phytomasse, taillis sclérophylle, chêne vert.

\section{Introduction}

La forêt domaniale de la Gardiole de Rians (Nord-ouest du département du Var) a fait l'objet, dans le cadre d'un contrat Piren (1) "Forêts sclérophylles " de nombreuses recherches interdisciplinaires associant les chercheurs de l'INRA, du C.N.R.S. et de l'Université.

Cette forêt acquise par l'Etat en 1937 a l'avantage de présenter une topographie contrastée conduisant à l'individualisation de milieux très différents dans un cadre géographique où dominent les calcaires compacts diaclasés ou non ; les colluvions et les grèzes (Callot, in Marsteau, 1979). Du point de vue climatique, la région est soumise au bioclimat subhumide d'Emberger.

Les peuplements forestiers sont constitués de taillis purs à Chêne vert sur crêtes, plateaux sommitaux exposés au Mistral et sur pentes fortes en adret ; de taillis à Chêne pubescent aux expositions Nord et sur colluvions de dépressions ainsi que sur pentes douces. Les chênaies mixtes à Chêne vert et à Chêne pubescent sont également fréquentes.

(1) Programme environnement du C.N.R.S. (1981-1983) réalisé sous la responsabilité de MM. les Professeurs Barbero, Quezel et Delabraze (Station de sylviculture méditerranéenne INRA, Avignon). 
Sur le taillis de Chêne vert de cette forêt dont on connaît bien le plan d'aménagement, des travaux antérieurs (Miglioretri. 1981. et 1983; Barbero \& Miglioret7i, 1984) ont déjà permis de comparer la structure horizontale ou densité des peuplements de Chêne vert en relation avec leur composition floristique dans diverses situations écologiques où croît cette essence. Deux ensemblıs phytoécologiques ont été distingués selon la nomenclature proposée par BARbero \& LoISEL (1983) pour la Provence : l'un réunit les chênaies sclérophylles denses des Quercetalia ilicis, l'autre regroupe les structures de chênaies à recouvrement faible ou moyen des Pistacio Rhamnetalia.

Par ailleurs, des études dendrométriques plu; poussées ont été engagées (MiglioRETTI, 1983) afin de proposer pour les taillis sclérophylles dont on connaît l'âge d'exploitation une méthodologie spécifique permettant d'appréhender leur structure horizontale, leur croissance et leur production en envisageant aussi le fonctionnement des cépées.

Dans cet article sont exposés les résultats relatifs à :

- la production des yeuseraies dans un systime de taillis,

- la croissance des tiges de Chêne vert, en diamètre et en hauteur, afin de déterminer les potentialités par la caractérisation d'un certain nombre de classes de fertilité.

Sept stations avaient été retenues pour l'étudé de la structure. L'inventaire descriptif du bouquet de taillis, qui correspond au grcupement végétal pris au sein de la formation étudiée en tenant compte des effets de marge, s'est fait pour les différentes stations sur des placettes dont la forme est polygonale car elle est la mieux adaptée aux structures rencontrées. La superficie des placettes varie de $70 \mathrm{~m}^{2}$ à $112 \mathrm{~m}^{2}$, et a été induite par la surface du bouquet de taillis lui-méme ainsi que par la stabilisation des données collectées par placeaux successifs. Les classes d'âge des placettes se répartissent de 10 à 65 ans ; seule la plus âgée est située en exposition Nord, les autres en situation Sud. Les altitudes s'échelonnent de $450 \mathrm{~m}$ à $570 \mathrm{~m}$.

\section{Matériel et méthodes}

Une étude préalable basée sur la réalisation cle relevés phytoécologiques selon des transects parallèles à la pente et en courbes de niveau a permis une connaissance écofloristique des peuplements en présence. Puis au sein du taillis de Chêne vert, l'échantillonnage s'est fait de façon stratifiée sur les bases de la composition floristique et en fonction du critère âge appréhendé par des documents O.N.F. concernant la répartition des essences et les passages présumés d'intervention sylvicole.

\subsection{Dispositif d'étude de la biomasse}

L'approche méthodologique relative à l'évaluation de la phytomasse du taillis sclérophylle a pris appui sur la méthode de l'analyse dimensionnelle et allométrique dite encore mixte (Pardé, 1980).

La phase destructive de l'étude r'a porté que sur deux stations de chênaies vertes situées en versant Sud près de la partie sommitale du "massif forestier " et placées dans la même classe d'âge sur le plan parcellaire. Cet âge n'a pu être vérifié avec certitude qu'ultérieurement. 
La phytomasse a été déterminée par coupe et pesée d'individus appartenant à une même cépée moyenne celle-ci étant définie à partir des données moyennes, regroupées par cépées, des données de l'inventaire établies pour chaque station. La cépée retenue est celle qui se rapprochait le plus des différentes valeurs moyennes (Surface terrière de la cépée, hauteur de la cépée, nombre de brins par cépée, nombre de brins supérieurs à $8 \mathrm{~cm}$ de circonférence à $1,30 \mathrm{~m}$ de hauteur, circonférence à $1,30 \mathrm{~m}$ et hauteur de ces derniers) en tenant compte des intervalles de confiance afin de s'assurer de la bonne représentation de la cépée choisie au regard du peuplement. Cette méthode de la "cépée moyenne» peut être rapprochée de celle de l'arbre de surface terrière moyenne. Les brins abattus (5 pour la station 1, 9 pour la station 2 soit au total 14) ont été débités en billons de $50 \mathrm{~cm}$ depuis leur base jusqu'à la découpe $2,5 \mathrm{~cm}$ de diamètre, pesés sur place pour établir leurs poids frais et à chaque niveau de découpe une rondelle a été prélevée pour en déterminer le poids sec. Tous les rameaux inférieurs à cette découpe ont été regroupés dans le houppier puis effeuillés. Une pesée fraîche séparée des rameaux et des feuilles a été effectuée, puis un sous-échantillon a été prélevé pour le poids frais puis sec. Les poids secs ont été obtenus après étuvage à 105 "C jusqu'à poids constant.

\subsection{Dispositif d'étude de la production ligneuse}

Ce sont 26 brins qui ont été abattus : deux brins dominants par station, plus ceux provenant sur deux stations de l'abattage total de la cépée moyenne, un des brins dominants leur appartenant. Ces brins ont servi pour l'étude de la production en volume et celle de la croissance en hauteur.

Les courbes de croissance en diamètre ont été effectuées, à partir de l'épaisseur moyenne des 20 derniers cernes, pour les brins dominants soit au total 14 tiges, sur la rondelle prélevée à $1,50 \mathrm{~m}$ tige abattue. Cette rondelle est peu différente de celle à $1,30 \mathrm{~m}$ définie sur pied, le débittage ne pouvant commençer réellement au sol.

L'épaisseur moyenne a été calculée à partir de la mesure selon trois directions (DE Martin, 1971 ; Munaut, 1979).

\section{Résultats}

L'analyse des résultats est basée sur le regroupement en données simples, par la méthode statistique classique, des différentes mesures effectuées lors de l'inventaire, afin d'en déduire des paramètres caractéristiques des populations étudiées mais aussi sur l'étude de relations existant entre ces paramètres.

\subsection{Poids secs. Tarifs phytomasse. Production de matière sèche}

La teneur en eau augmente depuis le tronc vers les rameaux et les feuilles, ainsi que le long du brin où la liaison est linéaire entre le niveau de prélèvement de la rondelle et son taux d'humidité.

La variabilité du pourcentage en eau le long du brin laisse supposer que la détermination du poids sec nécessite le prélèvement de ondelles à plusieurs niveaux (Auclair \& Metayer, 1980). Cependant, le poids sec pourrait être estimé à partir 
d'une seule rondelle. A cette fin, on peut déterminer les écarts atteints selon la méthode utilisée, par le rapport : $\underline{\mathrm{PS}-\mathrm{PS}_{\mathrm{r}}}$

PS : poids sec estimé à partir de toutes les rondelles prises sur le brin

$\mathrm{PS}_{\mathrm{r}}$ : poids sec estimé à partir d'une seule rondelle.

La rondelle choisie pour les calculs est celle située à $1 \mathrm{~m}$ car parmi les brins coupés, certains n'atteignent pas $1,50 \mathrm{~m}$ et il y aurait eu une perte d'information. Les résultats montrent que le prélèvement d'une seule rondelle entraîne pour la station située dans une zone à faible bilan hydrique une sous-estimation $(-4,6$ p. 100) et pour la station située dans une microdépression une sur-estimation $(+3,6$ p. 100). Le choix de la méthode est donc fonction de la précision voulue.

L'ensemble des données obtenues, tout en restant insuffisant, a permis d'établir une approche évaluative de la phytomasse.

Auparavant, il a été envisagé un test de comparaison sur les moyennes et une analyse de variance, sur les poids secs de deux stations où ont été coupées les cépées, pour tester si leur différence était uniquement due au hasard, ou alors s'il s'agissait de deux populations différentes (Lamotte, 1971). Les valeurs de $t$ et $F$ étant nettement inférieures à celles lues dans les tables correspondantes, on peut conclure que les différences observées ne sont pas imputables à autre chose que le hasard; pour cette raison, les données des deux stations ont été regroupées. Différentes corrélations ont alors été testées afin de déterminer l'équation de régression linéaire la mieux corrélée, qui pourra alors être retenue pour l'estimation de la biomasse des taillis sclérophylles.

C'est l'équation liant le poids sec du brin à sa circonférence à 1,30 $\mathrm{m}$ qui a été appliquée sur les deux stations à l'ensemble des tiges de la station, regroupées par classes de circonférence.

$$
P=\mathrm{aC}_{1.31 \mathrm{n})}+\mathrm{b}
$$

En effet, l'élévation au carré de la circonférence ou l'introduction d'un autre paramètre tel que la hauteur n'ayant pas amélioré le tarif de façon notable, nous avons privilégié le tarif le plus simple.

Par extrapolation, ont pu être déterminées:

- la phytomasse totale, puis celle des troncs, rameaux et feuilles,

- la production moyenne en tonnes de matière sèche par hectare et par an.

Cette production ne tient pas compte des petits brins puisqu'ils ont un diamètre inférieur à celui fixé comme limite de mesure et scruvent n'atteignent pas 1,30 m, ni des pertes par mortalité, ni du renouvellement des feuilles.

A partir des équations de régression établies pour le Chêne vert, a également été calculée la phytomasse des autres espèces ligneuses présentes sur ces stations.

Il est évident que la généralisation, à d'autres essences, des tarifs concernant le Chêne vert peut créer un biais. C'est pourquoi ces résultats ne sont donnés qu'à titre purement indicatif.

Ce même tarif a aussi été appliqué à deux autres stations situées en même versant et de même classe d'âge, et ce en prenant aussi appui sur les résultats des courbes de croissance qui situent ces stations dans la même classe de fertilité. 
On notera la part importante jouée par les essences autre que le Chêne vert dans la phytomasse des différentes stations. L'ensemble de ces résultats est porté dans le tableau 1. La production moyenne annuelle en matière sèche, toutes essences et stations confondues, varie entre $3 \mathrm{t} / \mathrm{ha} /$ an et $3,6 \mathrm{t} / \mathrm{ha} /$ an pour des peuplements de 25 . 30 ans.

\subsection{Volume}

Le calcul du volume a été réalisé par la méthode des billons successifs auxquels est appliquée une formule de cubage (PARdÉ, 1961 ; Bouchon, 1974).

\section{TABLEAU 1}

Production moyenne de matière sèche en tonnes par hectare (t/ha) sur des peuplements âgés de 25 à 30 ans.

Mean production of dry matter (t/ha) for twenty to thirty year old stands.

\begin{tabular}{|c|c|c|c|c|c|c|c|}
\hline \multicolumn{2}{|c|}{$\begin{array}{l}\text { Stations } \\
25-30 \text { ans }\end{array}$} & $\begin{array}{l}\text { "Tronc } \\
\text { t/ha }\end{array}$ & $\underset{\text { t/ha }}{\text { Rameaux }}$ & $\begin{array}{c}\text { « Feuilles } \\
\text { t/ha }\end{array}$ & $\begin{array}{l}\text { «Total } \\
\text { t/ha }\end{array}$ & $\begin{array}{c}\mathrm{QI}+ \\
\text { autres }\end{array}$ & $\begin{array}{l}\% \text { essences autres que } \\
\text { le QI dans le total }\end{array}$ \\
\hline \multirow{2}{*}{1} & QI & 65 & 14 & 5 & 8.1 & \multirow{2}{*}{$\begin{array}{l}90,4 \\
\text { t/ha }\end{array}$} & \multirow{2}{*}{$7,6 \%$} \\
\hline & A & 5 & 1 & 0,4 & 6,4 & & \\
\hline \multicolumn{2}{|l|}{2} & 78,6 & 16 & 6,4 & 101 & $\begin{array}{l}101 \\
\text { t/ha }\end{array}$ & $0 \%$ \\
\hline \multirow{2}{*}{3} & QI & 57 & 11,5 & 4,6 & 93 & \multirow{2}{*}{$\begin{array}{c}90,8 \\
\text { t/ha }\end{array}$} & \multirow{2}{*}{$19,6 \%$} \\
\hline & $\mathrm{A}$ & 18,6 & 3 & 1,1 & 19,8 & & \\
\hline \multirow{2}{*}{6} & QI & 52 & 10,4 & 8 & 65,4 & \multirow{2}{*}{$\begin{array}{l}76,6 \\
\text { t/ha }\end{array}$} & \multirow{2}{*}{$14,6 \%$} \\
\hline & $\mathrm{A}$ & 8,5 & 1,9 & 0,7 & 11,2 & & \\
\hline
\end{tabular}

QI : Quercus ilex (Chêne vert) / Quercus ilex (Holly oak). A : Autres ligneux / Others woods.

Le volume a été calculé pour les 26 tiges abattues, à partir de la formule de Smalian. Celle-ci est d'un emploi aisé et sa précision a été augmentée en prenant des billons de $50 \mathrm{~cm}$. Le calcul du volume tige, pris ici dans le sens "objet physique " représenté par la tige, a été réalisé à la découpe $2,5 \mathrm{~cm}$ de diamètre qui est le diamètre limite que nous avons retenu afin d'effectuer l'inventaire dendrométrique des brins compte tenu de la grande représentativité des brins de faible diamètre.

Pour ce site, c'est l'équation liant le volume au carré de sa circonférence à $1,30 \mathrm{~m}$ qui a été retenue comme tarif $(r=0,977)$. L'équation est alors appliquée à la valeur centrale de chaque classe de l'histogramme de fréquence des circonférences préalablement établi lors de l'inventaire dendrométrique. Ainsi, par extrapolation à l'effectif des classes considérées, on peut en déduire le volume des tiges de la placette étendues ensuite à l'hectare (tabl. 2).

Ce tableau comporte les résultats de six placettes seulement car pour la placette $\mathrm{n}^{\circ} 7$ âgée de 5-10 ans les paramètres dendrométriques - circonférence, hauteur — ont été pris à $0,10 \mathrm{~m}$ car à $1,30 \mathrm{~m}$ le diamètre limite retenu à savoir $2,5 \mathrm{~cm}$ était rarement atteint. De ce fait les calculs ne pouvaient être réalisés à partir du tarif retenu. 


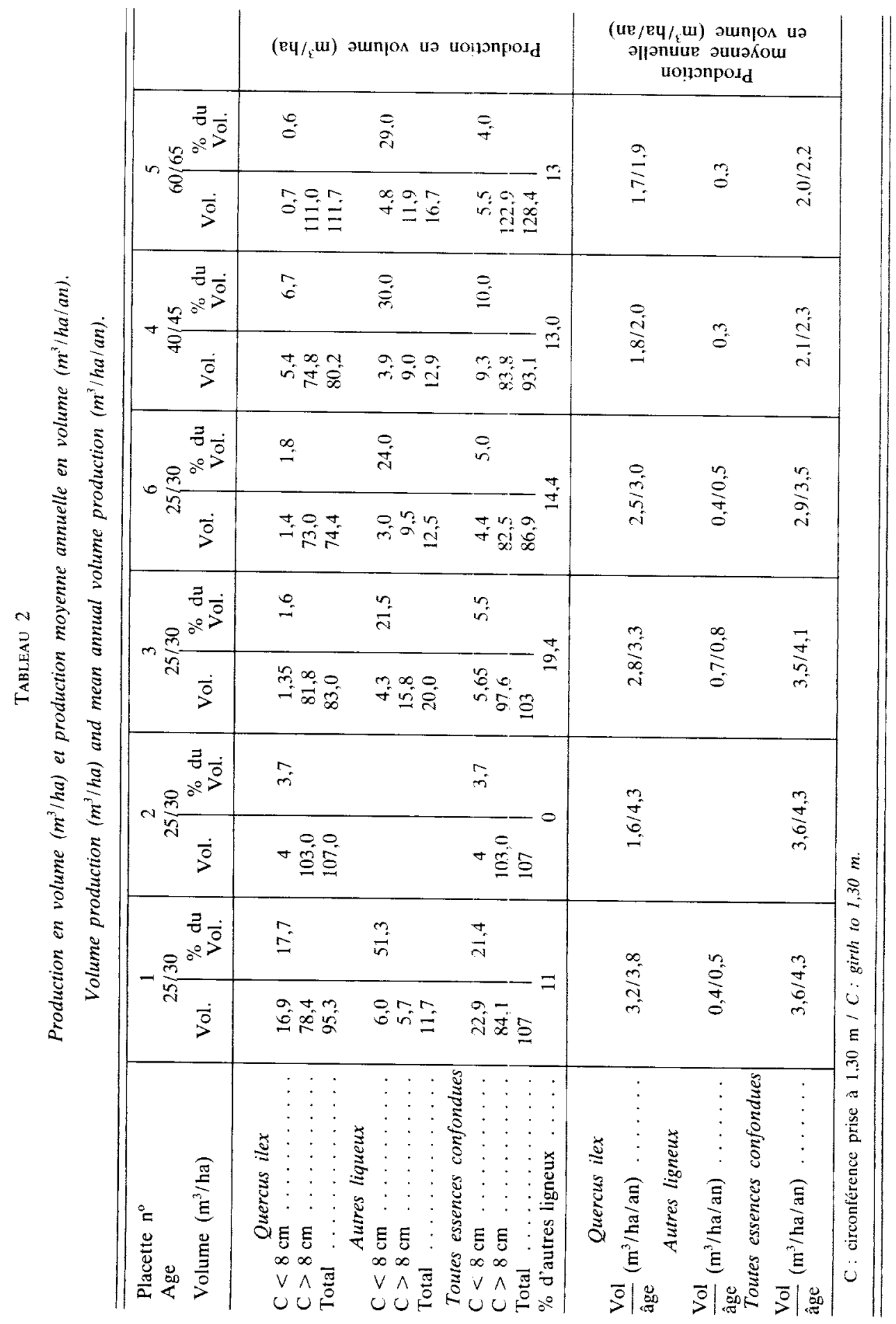


Les valeurs trouvées correspondent à des données maximales compte tenu du dispositif expérimental qui n'intègre pas les trouées.

\subsection{Production moyenne annuelle}

Calcul par le tarif $V=a C_{1,30 \mathrm{~m}}^{2}+b$

La production a été calculée en se référant à l'âge du brin dominant. Cette production moyenne annuelle sur l'ensemble des stations varie entre $1,9 \mathrm{~m}^{3} / \mathrm{ha} / \mathrm{an}$ et $4,3 \mathrm{~m}^{3} / \mathrm{ha} / \mathrm{an}$ avec une moyenne de $3 \mathrm{~m}^{3} / \mathrm{ha} / \mathrm{an}$, pour le Chêne vert dans les aires échantillonnées.

Le mème tarif a été appliqué aux autres essences. De ce fait, toutes essences confondues, la production moyenne s'élève en moyenne à $3,4 \mathrm{~m}^{3} / \mathrm{ha} / \mathrm{an}$, les extrêmes étant $2,1 \mathrm{~m}^{3} / \mathrm{ha} /$ an et $4,3 \mathrm{~m}^{3} / \mathrm{ha} / \mathrm{an}$ dans ces mêmes stations.

La production moyenne annuelle des différentes placettes est portée au tableau 2.

Ce tableau met en évidence dans la production :

- l'importance des brins de faible circonférence (inférieure à $8 \mathrm{~cm}$ à $1,30 \mathrm{~m}$ ) dénombrés mais non mesurés effectivement puisqu'ils n'avaient donc pas le diamètre limite que nous avions fixé. De ce fait, par la suite il a été considéré que ces brins avaient en moyenne $4 \mathrm{~cm}$ de circonférence, valeur qui correspond au centre de classe dans laquelle ils ont été regroupés ;

- la participation des brins autres que le chêne vert.

On constate que la station 2, située en talweg et sur pente, réalise la plus forte production annuelle moyenne et ce, uniquement avec le Chêne vert ; de plus, cette production est constituée en grande partie par des brins de circonférence supérieure à $8 \mathrm{~cm}$. Cette station semble être celle où l'adéquation conditions de milieu-croissance Chêne vert est la mieux réalisée.

\subsection{Courbes de croissance en hauteur et classes de fertilité}

Les classes de fertilité ont été établies à partir des deux brins dominants prélevés par station. L'indice de productivité a été défini par la base de la hauteur atteinte à l'âge de 25 ans. Pour cela, on a cherché à ajuster les courbes de croissance tracées, à un modèle mathématique. Le modèle retenu est la forme :

$$
\begin{array}{ll}
H=b \times A^{\prime \prime} & r=0.826 \\
H: \text { hauteur }(m) & n=1.35 \\
A: \text { age (ans) } &
\end{array}
$$

La hauteur dominante moyenne atteinte à 25 ans calculée à partir de l'équation est égale à $4.65 \mathrm{~m}$.

Les stations étudiées en Gardiole de Rians se répartissent en 4 classes de fertilité à 25 ans (fig. 1). 


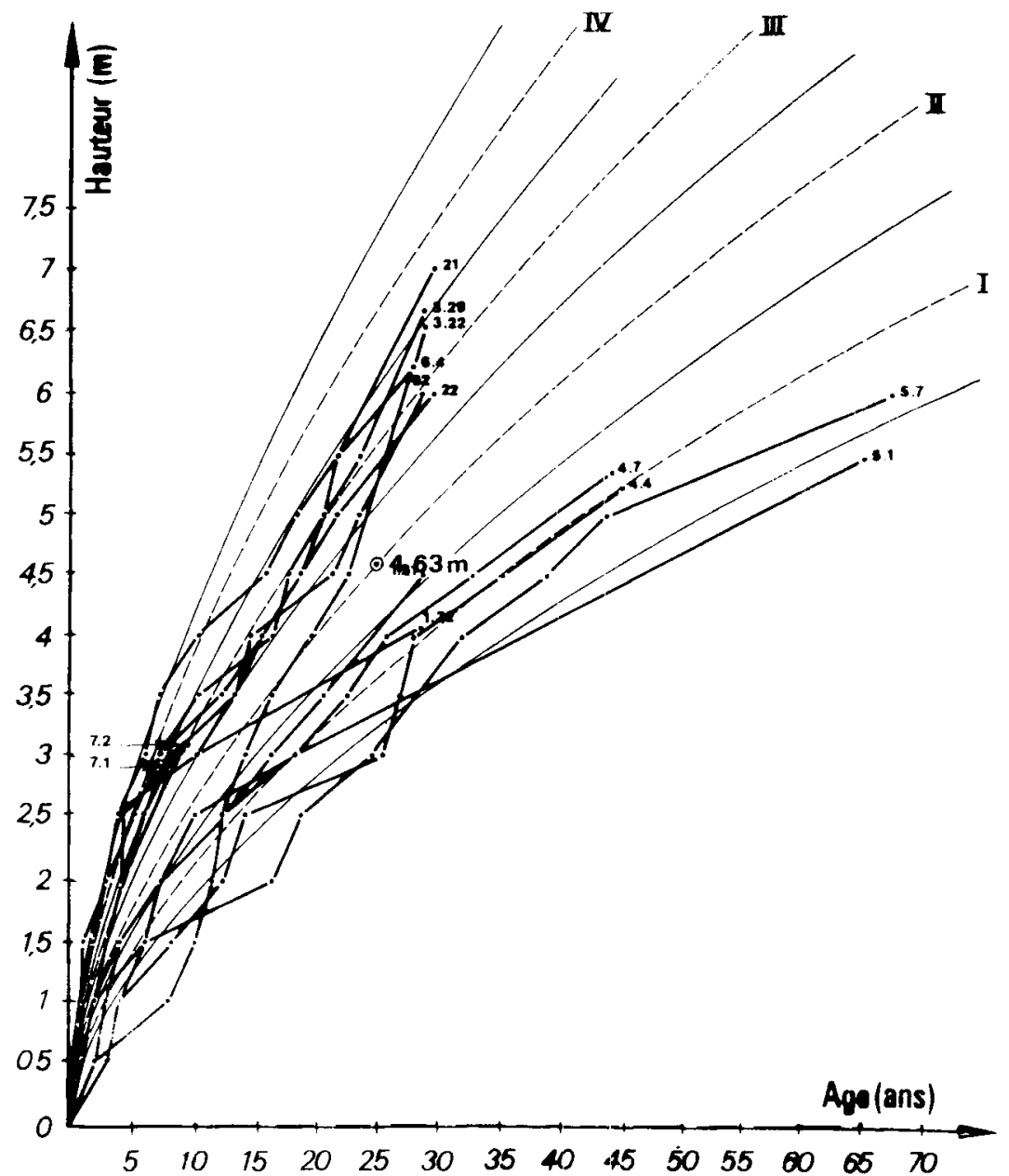

FIG. 1

Courbes de croissance des brins dominants replacés dans leurs classes de fertilité. Growth curves of the dominant shoots in their fertility classes.

Signification des chiffres faisant suite aux courbes / Meaning of the number following the curves : ex : $3.22=3:$ numéro de la station / station number. 22 : numéro de la cépée / stump number. 


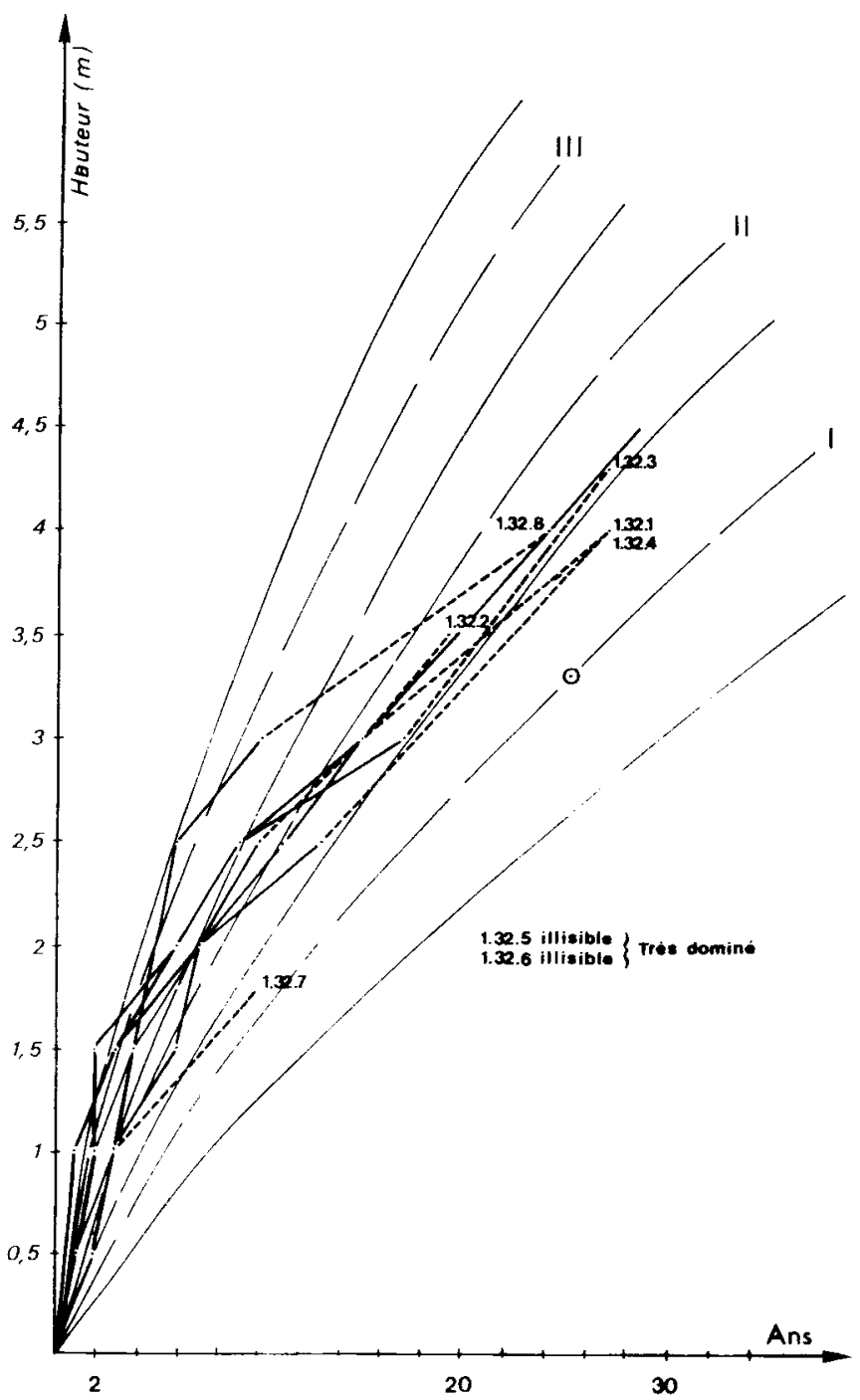

FIG. 2

Courbes de croissance des brins d'une même cépée replacés dans leurs classes de fertilité (station $n^{\prime \prime}$ l).

Growth curves of the shoots on the same stump in their fertility classes (station $n^{\prime \prime} 1$ ). 
Par la suite, on a reporté sur le graphe. les courbes de croissance en hauteur des brins prélevés sur les cépées afin de voir à quel niveau de fertilité ils se situaient. Il semblerait que les brins d'une même cépée se situent dans la même classe de croissance que le brin dominant. Une réserve est à apporter à cette conclusion en particulier dans

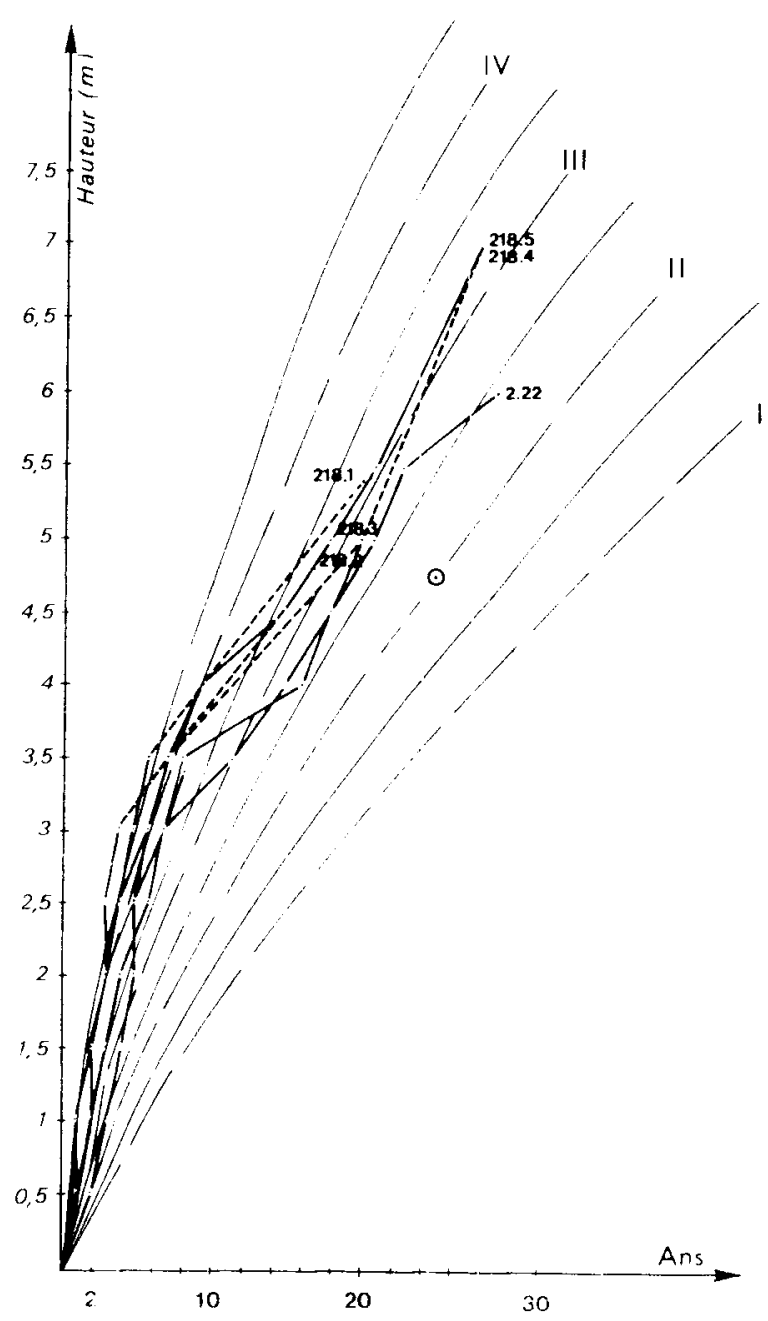

FIC. 3

Courbes de croissance des brins d'une même cêpée replacés dans leurs classes de fertilité (station $n^{\prime \prime} 2$ ).

Growth curves of the shoots on the same stump in their fertility classes (station $n^{\circ} 2$ ).

Signification des chiffres faisant suite aux courbes /

Meaning of the number following in the curves:

ex : $1.32 .1=1$ : numéro de la station / station number.

32: numéro de la cépée / stump number.

1 : numéro du brin sur la cépée/ shoot number on the stump. 
les taillis se développant dans de mauvaises conditions stationnelles où les brins dominés, en général les plus jeunes, n'appartiennent pas à la même classe de croissance. Dans ce dernier cas, la sélection naturelle des brins s'opère plus lentement et la disparité d'âge observée implique que certains brins dominés sont situés dans une classe de fertilité inférieure ; lors du vieillissement du taillis, ces brins seront probablement éliminés (fig. 2 et 3 ).

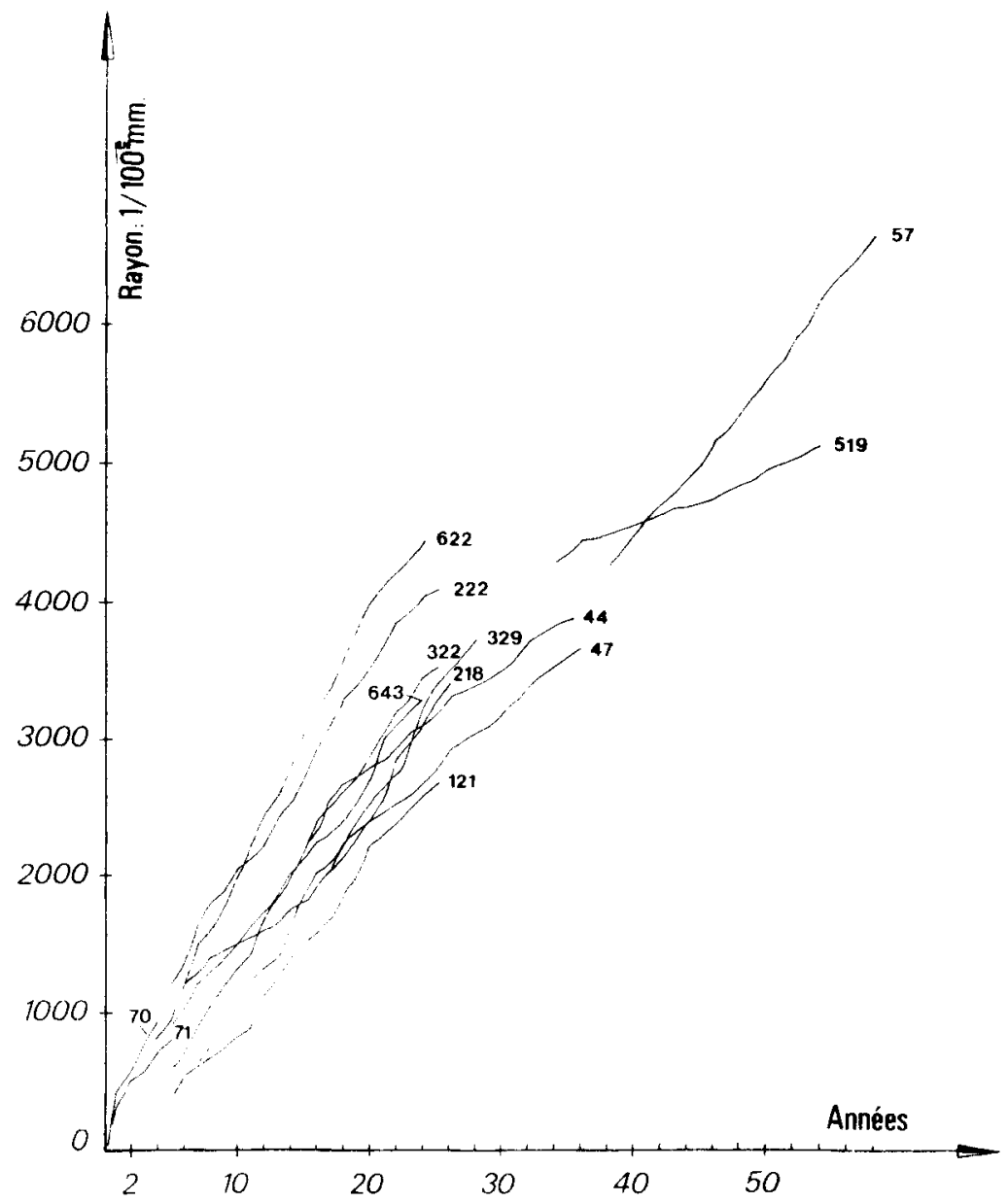

FIG. 4

Courbe de croissance sur le rayon.

Growth curves on the radius.

Signification des chiffres faisant suite aux courbes / Meaning of the number following in the curves: $\mathrm{cx}: 6.43=6:$ numéro de la station / station number . 43 : numéro de la cépée / stump number. 
On peut toutefois s'interroger sur la meilleure façon d'établir les classes de fertilité :

- celles basées sur les courbes de croissance supposent un nombre restreint de brins prélevés et des données ponctuelles. Cependant la connaissance de la croissance rend compte du dynamisme d'un peuplement. Elle permet ainsi de visualiser lis éventuels changements de classe de fertilité au cours du temps. L'évolution du peuplement, traduite par des modifications au sein des cépées, peut s'exprimer au niveau de la croissance des brins,

- elles peuvent être aussi établies à partir de l'âge et de la hauteur du brin dominant. L'inventaire peut alors être très élargi mais limite les renseignements sur l'adéquation conditions du milieu-évolution du peuplement. Il y aurait donc occultation d'informations en ce sens que l'on peut se demander si ce brin a toujours été dominant.

\subsection{Courbes de croissance sur le rayon}

Pour chaque rondelle, la courbe de croissance annuelle pour les 20 dernières années a été tracée, mais l'origine de chaque courbe tient compte de la croissance durant les années précédentes (fig. 4).

Par la suite, les différentes portions de courbe ont été ajustées à un modèle mathématique. Pour les stations étudiées en Gardiole de Rians, la courbe de croissance suit un modèle puissance, d'équation :

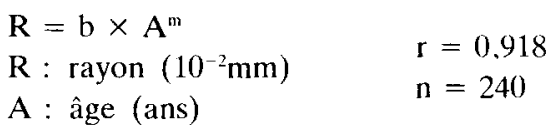

Il faut cependant formuler quelques remarques:

- dès la $26^{\text {e }}$ année nous ne possédons que deux valeurs moyennes, ce qui introduit sans doute un biais à partir de ces années,

- de plus, à partir de la $37^{\mathrm{e}}$ année, les valeurs ont été établies sur la base de données lues sur des brins situés dans une mauvaise classe de fertilité entrainant un amortissement trop précoce de la courbe ; toutefois, on peut penser qu'il y a effectivement un ralentissement de la croissance en épaisseur des tiges au cours du temps.

\section{Conclusion}

L'étude dendrométrique des taillis de Chêne vert (Miglionetri, 1983), peu étudiée ici, a permis de tester sur des peuplements sclérophylles les méthodologies proposées par PARdé et utilisées, il est vrai, dans la majeure partie des cas en futaie.

Dans toutes les stations échantillonnées, on a toujours pu observer un nombre important de brins de faible diamètre. En effet, lors de l'inventaire dendrométrique ont été compté d'une part le nombre de brins et d'autre part le nombre de brins mesurés en hauteur et circonférence, cette mesure n'étant effectuée que pour les brins ayant un diamètre supérieur à $2,5 \mathrm{~cm}$ à $1,30 \mathrm{~m}$ de hauteur. Le taux de brins non mesurés par 
rapport au nombre de brins total varie de 10 p. 100 à 60 p. 100 selon les placettes étudiées. Ces valeurs auraient donc été encore plus élevées si nous avions pris comme référence le niveau de découpe retenu couramment en foresterie classique c'est-à-dire la découpe bois-fort $(7 \mathrm{~cm}$ de diamètre à $1,30 \mathrm{~m}$ de haut $)$. Les brins de faible diamètre ont donc en fait une part importante dans la production totale ; la production en volume bois-fort ou en biomasse ne représente alors dans ces structures sclérophylles qu'un faible pourcentage de la production totale.

En outre, il a été démontré que dans le taillis sclérophylle le poids sec des brins était corrélé linéairement avec leur circonférence à $1 \mathrm{~m} \mathrm{30}$. De même, l'estimation de la teneur moyenne en eau du brin pourrait se faire en ne prélevant qu'une seule rondelle, mais il conviendrait alors de tester dans chaque placette, au moins sur un faible nombre de représentants, l'erreur commise dans le calcul de la phytomasse. Par contre, la variation du taux d'humidité dans les parties constitutives du brin implique la collecte d'échantillons séparés : tige, rameaux, feuilles.

Pour l'évaluation du volume plusieurs remarques peuvent être formulées :

- son approche par l'utilisation d'un tarif établi à partir de deux brins dominants, fait que l'on tend à surestimer les stations les moins fertiles et à sous-estimer les meilleures. Cependant cette méthode présente l'avantage d'être facilement généralisable ;

- son estimation par l'intermédiaire du volume d'une cépée moyenne est sans doute plus représentative de la station, mais elle est d'un emploi plus lourd.

On pourrait aussi envisager un échantillonnage stratifié mais la précision du tarif, compte tenu de la faible valeur commerciale de ces peuplements, ne semble pas justifier un investissement aussi important.

L'étude de la croissance a montré que les brins d'une même station, mais d'âge différent, semblaient suivre la même loi de croissance que les brins dominants, du moins dans les stations écologiquement favorables. Dans ces stations en effet, la sélection naturelle intervient plus rapidement dans le temps et la compétition pour la lumière est moins marquée.

Cette recherche a permis de rendre compte au niveau des placettes d'échantillonnage et dans toutes les situations observées, des phénomènes de compétition dont les causes sont multiples:

- concurrence entre brins d'une même cépée, comme le soulignent les paramètres dendrométriques (circonférence, hauteur). Les variations marquées entre ces caractéristiques dendrométriques pour des brins appartenant à une même cépée traduisent des différences d'affranchissement mais aussi un fonctionnement particulier de la cépée sclérophylle qui émet des brins à des périodicités successives, une cépée pouvant porter des brins d'âge différent comme nous l'avons observé lors de l'analyse de tige ;

- concurrence entre cépées en fonction de leur distribution spatiale, le doublement de la surface d'inventaire ne doublant pas le nombre de cépées. On retrouve là une des caractéristiques majeure des milieux méditerranéens : l'hétérogénéité ;

- compétition interspécifique liée à la présence du Chêne blanc ou à d'autres essences caducifoliées.

L'augmentation de la hauteur moyenne des brins dominants des taillis de même âge et de même exposition mais situés dans des conditions stationnelles différentes illustre les potentialités des stations. 
Sur une même cépée, l'observation de brins d'âges différents montre que dans le taillis de Chêne vert le dépressage naturel s'opère jusqu'à un âge moyen assez élevé. Cependant. la sélection naturelle des brins intervient d'autant plus tôt, pour des taillis de même âge. que les conditions géopédologiques de la station sont meilleures.

Compte tenu de toutes ces particularités la question qui se pose est de savoir comment et quand faire une intervention sylvicole (dépressage, exploitation...), et quelles en seraient les conséquences.

Y aurait-il transfert de croissance, notamment en diamètre sur les brins dominants laissés? L’apparition de nouveaux rejets sur la cépée ne conduirait-elle pas à un épuisement plus rapide de la souche?

Dans le cas où il y aurait transfert, il conviendrait de prendre en compte. làge du taillis, la sélection naturelle des brins et la situation éco-stationnelle, afin d’intervenir à des périodes plus favorables pour obtenir le meilleur report sur les brins laissés.

On comprend dès lors toutes les difficultés à gérer un tel écosystème à sclérophylle. De plus, le coût de telles opérations serait-il rentabilisé par une production accrue en qualité et en quantité ?

Reçu le 14 octobre 1985.

Accepté le 10 octobre 1986.

\author{
Summary \\ Contribution to the study of schlerophyllous coppice production \\ in the Gardiole of Rians forest (Var)
}

\begin{abstract}
This article deals with a method of study for schlerophyllous coppice volume and biomass production in the Gardiole de Rians (Var, France) forest, as well as height and diameter growth in Holly Oak (Quercus ilex) shoots in order to determire a certain number of fertility classes. By considering all the shoots on a stump, coppice production level can be hetter expressed and the fonction of periodic stem production on Holly (lak stumps better understood. Problems of interspecific competition for field ressource use can also be dealt with. This study also considers some mathematical models useful in the analysis of various production levels and height and diameter growth in Holly Oak stems.
\end{abstract}

Key words : Growth, production, phytomass, schlerophyllous coppice, holly oak.

\title{
Références biblioģraphiques
}

Auclair D.. Mrisyer S., 1980. Méthodologie de lévaluation de la biomasse aćrienne sur pied et de la production en biomasse des taillis. Acta Oecol., Oecol. Plant, 1 (4). 357-376.

Barbero M., Loisel, 1983. Les chênaies vertes du Sud-Est de la France méditerranéenne. Valeurs phytosociologiques, dynamiques et potentielles. Phyrocoenologia, 11 (2), 225-244. 
Barbero M., Migljoretri F., 1984. Etude comparée de la densité des peuplements de Chêne vert en relation avec leur composition floristique : application à la forêt domaniale de la Gardiole de Rians (Var). Ecologia mediterrunea, Tome X (Fasc. 1-2), $205-230$.

Bouchon J., 1974. Les tarifs de cubage. INRA, C.N.R.F., E.N.G.R.E.F., Nancy, 57 p. + annexes.

De. MARTin P., 1970. Les anneaux de croissance des arbres : dendroclimatologie et dendrochronologie. Revue géographique de l'Est, 3-4, 279-287.

Lamotte M., 1971. Initiation aux méthodes statistiques en biologie. Masson et Cie, $2^{c}$ éd., $141 \mathrm{p}$.

Marsteau C., 1979. Structure, dynamique et mise en valeur forestière d'une zone à Chêne pubescent. Mémoire Ing. E.N.I.T.E.F.. Station de sylviculture forestière méditerranćenne, 92 p. + annexes.

MigLioretTI F., Roche B., 1981. Etude cadastrale et premières approches méthodologiques pour l'évaluation de la phytomasse des taillis de Chêne vert en forêt domaniale de la Gardiole de Rians. Rapport D.E.A., Saint-Jérôme, Marseilte.

Miglioretri F., 1983. Phytoécologie des peuplements à Quercus ilex L. et Quercus pubescens Willd. en Gardiole de Rians (Var). Approche méthodologique pour évaluer la phytomasse des taillis de Chêne vert. Thèse $3^{*}$ cycle, Saint-Jérôme, Marseille, 77 p. + annexes.

Munaut A.V., 1979. La dendrochronologie et les problèmes de géographie historique. Extrait de Source de la géographie historique en Belgique. Actes du Colloque de Bruxelles, 25-27, IV, $471-486$.

Munaut A.V., 1979. La dendrochronologic. Bulletin de l'Association française pour l'étude du Quaternaire, 1-2, 65-74.

Pardé J., 1961. Dendrométrie. Nancy Edit. Ecol. Nat. Eaux et Forêts, 350 p.

PARdÉ J., 1980. Les biomasses forestières. INRA, C.N.R.F., Nancy, 54 p. 